\title{
When Blue is Larger than Red: Colors Influence Numerical Cognition in Synesthesia
}

\author{
Roi Cohen Kadosh' ${ }^{1}$, Noam Sagiv ${ }^{2}$, David E. J. Linden ${ }^{3,4}$, \\ Lynn C. Robertson ${ }^{5,6}$, Gali Elinger ${ }^{1}$, and Avishai Henik ${ }^{1}$
}

\begin{abstract}
In synesthesia, certain stimuli ("inducers") may give rise to perceptual experience in additional modalities not normally associated with them ("concurrent"). For example, color-grapheme synesthetes automatically perceive achromatic numbers as colored (e.g., 7 is turquoise). Although synesthetes know when a given color matches the one evoked by a certain number, colors do not automatically give rise to any sort of number experience. The behavioral consequences of synesthesia have been documented using Stroop-like paradigms, usually using color judgments. Owing to the unidirec-
\end{abstract}

\section{INTRODUCTION}

Synesthesia is a condition in which sensory experiences (e.g., sounds, tastes) or concepts (e.g., words, numbers, time units) automatically evoke additional percepts (most commonly color) (Sagiv, 2004). Synesthetic experiences occur both between (e.g., colorphoneme) and within (color-grapheme) sensory modalities (Grossenbacher \& Lovelace, 2001). Whereas the initial study of synesthesia was based on introspection (Galton, 1880), this approach has been supplemented by objective methods in recent years. One of the most powerful paradigms for investigating synesthesia employs conflict situations like the Stroop task (Stroop, 1935). In the classical Stroop paradigm, normal participants are presented with colored words. The participants' task is to name the color of the ink while ignoring the word's meaning. The classic finding is that normal participants encounter difficulties in naming the ink color of a word when it is incongruent with its meaning (e.g., BLUE printed in red) relative to when it is congruent with the meaning (e.g., BLUE printed in blue). In contrast, if the participants are asked to read the printed word while ignoring the ink color, (i.e., reverse Stroop), they usually do not encounter difficulties with incongru-

\footnotetext{
${ }^{1}$ Ben-Gurion University of the Negev, ${ }^{2}$ University College London, ${ }^{3}$ University of Wales, ${ }^{4}$ Goethe University, ${ }^{5}$ University of California-Berkeley, ${ }^{6}$ Neurology Research Services, Veterans Affairs Medical Center, Martinez, CA, USA
}

tional nature of the synesthetic experience, little has been done to obtain performance measures that could indicate whether bidirectional cross-activation occurs in synesthesia. Here it is shown that colors do implicitly evoke numerical magnitudes in color-grapheme synesthetes, but not in nonsynesthetic participants. It is proposed that bidirectional coactivation of brain areas is responsible for the links between color and magnitude processing in color-grapheme synesthesia and that unidirectional models of synesthesia might have to be revised. ent trials. These findings suggest that in skilled readers, reading words is more automatic than is naming colors (MacLeod, 1991).

Similarly, when synesthetes are asked to name the color in which a grapheme is presented on the screen, congruency with the synesthetic color affects performance. Specifically, when the synesthetic color and the display color are incongruent, synesthetes are slower to name the color, relative to when the two colors match (Dixon, Smilek, \& Merikle, 2004; Palmeri, Blake, Marois, Flanery, \& Whestsell, 2002; Mattingley, Rich, Yelland, \& Bradshaw, 2001; Dixon, Smilek, Cudahy, \& Merikle, 2000; Mills, Boteler, \& Oliver, 1999; Odgaard, Flowers, \& Bradman, 1999). This interference effect indicates automatic processing of the concurrent experience, albeit not to the same degree in all synesthetes (Dixon et al., 2004). Although attention to the evoking stimulus does modulate the synesthetic Stroop effect (e.g., Sagiv \& Robertson, 2004), synesthetic experience is otherwise automatic and not subject to conscious control (e.g., Palmeri et al., 2002; Mills et al., 1999; Cohen Kadosh \& Henik, in press).

Defined in experiential terms, synesthesia is essentially unidirectional. Thus, digits may evoke colors, but the opposite pattern is not reported. Although synesthetes can voluntarily retrieve the numbers that normally evoke given colors, the process does not appear to be automatic and numbers are not experienced in any vivid form. The idea that synesthesia is essentially unidirectional has indeed been advocated by Mills et al. (1999) 
and has not been challenged (although some theories of synesthesia remain agnostic about the possibility of bidirectional interactions). Accordingly, the majority of behavioral studies of color-grapheme synesthesia measuring Stroop-like performance examined the interference induced by the grapheme in the color domain. In addition, the color of the synesthetic perception can interfere with the processing of the physical color of the grapheme (Dixon et al., 2004). In contrast, the perceived color does not interfere with grapheme processing. Nevertheless, a small number of studies suggest that synesthesia may have consequences for the processing of the inducing stimuli as well (Palmeri et al., 2002; Ramachandran \& Hubbard, 2001a; Smilek, Dixon, Cudahy, \& Merikle, 2001). It is central for our understanding of synesthesia whether the unidirectional nature of synesthetic experience (e.g., digits inducing the experience of color, but not vice versa) implies that the underlying cognitive and neural mechanisms (as well as the behavioral manifestations of these) are unidirectional too.

We developed a modified size congruity paradigm to examine whether colors influence numerical magnitude in color-grapheme synesthesia. It is important to note that none of our synesthetes reported that colors evoked numbers in their daily life experience, consistent with other reports on color-grapheme synesthesia.

In the classic size congruity paradigm, the physical size of a digit and the numerical value are orthogonally varied. Although participants are asked to refer to the numerical value and ignore the physical dimension, size is processed automatically and affects performance (e.g., Henik \& Tzelgov, 1982). For example, when the pair 24 is presented, processing of the numerical information is facilitated if the numerically larger digit is also physically larger (e.g., 2 4). In contrast, if the numerically smaller digit is physically larger (e.g., 2 4) interference occurs, and processing of the numerical information is slowed. Performance is also affected by the magnitude distance effect, with a faster comparison process for larger distances between two stimuli (Moyer \& Landauer, 1967). For example, if the numerical distance is large, the chances for the irrelevant physical size to interfere with numerical processing will be small. In addition, the distance effect along the irrelevant dimension (i.e., physical size) also affects the performance. For example, a large distance along the (irrelevant) dimension of physical size will be processed relatively fast and thus will be more likely to interfere with the (relevant) numerical dimension (Schwarz \& Ischebeck, 2003).

In this study, we replaced the irrelevant dimension of physical size with that of color. Participants were asked to decide which one of two digits was numerically larger and to ignore the color dimension. In the normal population, colors are not subject to ordinal scaling, but we hypothesized that colors may indicate ordinal scaling in synesthesia. This might be due to abnormal connections between the color and number systems of the brain or to learning of digit-color associations (Elias, Saucier, Hardie, \& Sarty, 2003). To test the latter possibility, we trained a learning group, which studied the digit-color association to a high level of expertise. We defined the color distance as the difference between the digits that induce these two colors for each synesthete. For example, the two colors evoked by the digits 2 and 7 represent a color distance of five units. In participants with synesthesia, we expected facilitation of performance similar to the effect of an irrelevant physical size whenever the colors indicated a larger distance than the relevant numerical values (e.g., the digits 4 and 5 printed in the colors induced by 2 and 7, respectively). In contrast, if colors do not induce quantity or magnitude, there should be no facilitatory effect of color on the time taken to make a magnitude judgment. This prediction departs from classical unidirectional models of synesthesia, which would predict only interference (or no effect at all) whenever the colors do not match the digits, but no facilitation.

Previous studies have shown that in nonsynesthetes, numbers and colors, unlike numbers and physical magnitude, do not interfere (Lammertyn, Fias, \& Lauwereyns, 2002; Fias, Lauwereyns, \& Lammertyn, 2001). Therefore, Fias and colleagues (2001) suggested that the interference between two dimensions is determined by the degree of neural overlap of structures dedicated to processing of specific mental operations. Although there is a great deal of overlap between the processing of numerical and physical magnitudes, for example, at the level of the parietal lobe (Pinel, Piazza, Le Bihan, \& Dehaene, 2004; Fias, Lammertyn, Reynvoet, Dupont, \& Orban, 2003; Cohen Kadosh et al., 2005), the same might not be true for numbers and color in nonsynesthetic individuals. In synesthetes, however, abnormal or abnormally preserved connections might exist between the number processing systems of the parietal lobe and the color areas in the parietal lobe (Cohen Kadosh \& Henik, in press) or the temporal lobe (Ramachandran \& Hubbard, 2001a, 2001b). If we can show that colors induce magnitude, this will suggest that these connections are bidirectional.

We tested two synesthetes with color-grapheme synesthesia, who perceived color whenever they see a digit. Both synesthetes describe the color as been projected "out there" on the digit itself (i.e., projector type, Dixon et al., 2004). Pairs of digits with a small numerical distance (e.g., 4-5) were used to create two conditions: (1) large color distance, when the two digits appeared in colors that indicated (for the individual synesthete) a larger distance than the numerical distance of the digit pair (e.g., the digit 4 appeared in the color associated with the digit 2, whereas the digit 5 appeared in the color associated with the digit 7), and (2) matched color, when each of the two digits appeared in the colors that corresponded to these digits for the individual synes- 


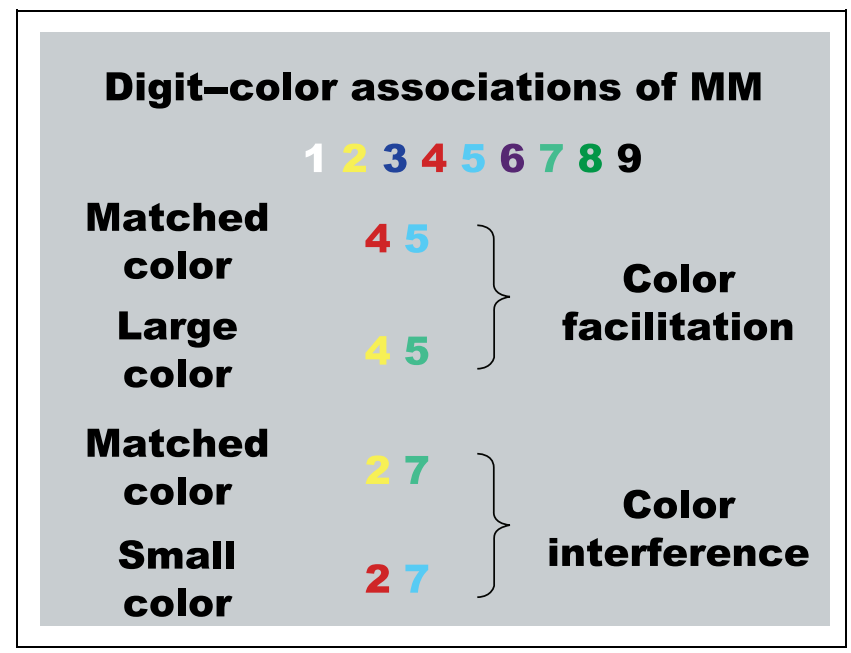

Figure 1. An example of M.M.'s color-grapheme association and the experimental conditions.

thete. For example, if the digit 4 was experienced as red, it appeared in red, and if 5 was experienced as blue, it appeared in blue. Hence, in this condition, the distance indicated by the colors matched the numerical distance (i.e., distance of one unit). These conditions were compared to examine if the irrelevant larger color distance facilitated numerical comparisons while the numerical distance is kept constant. Pairs of digits with a large numerical distance (e.g., 2-7) were used to produce two other conditions: (1) small color distance, when the two digits appeared in colors that indicated (for the individual synesthete) a smaller distance than the numerical distance of the digit pair (e.g., the digit 2 appeared in the color that was associated with the digit 4 , whereas the digit 7 appeared in the color associated with the digit 5, i.e., color distance of one unit), and (2) matched color, each of the two digits appeared in the colors that each synesthete experiences when presented with these digits (e.g., number and color distance of five units). These conditions were compared to examine whether the smaller (irrelevant) color distance interfered with numerical comparisons (while maintaining a constant numerical distance). We did not reverse the matching to induce interference at the response level (i.e., the digit 2 appeared in the color that was associated with the digit 5 , whereas the digit 7 appeared in the color associated with the digit 4) because this would have limited our ability to infer quantitative effects of color distance. In such a condition, one cannot separate interference due to the magnitude evoked by color from that due to conflict in the response initiation.

We compared the synesthetes' performance to two naïve control groups. In addition, we recruited six nonsynesthetic controls that formed the learning group. The learning group was extensively trained on color-digit association. This was carried out to examine the possibility that synesthetic performance is because mere learning (cognitive) association between digits and colors. Figure 1 illustrates the experimental conditions.

\section{RESULTS}

Data of the synesthetes and the control participants were analyzed separately.

\section{Synesthetes}

Both synesthetic participants (M.M. and A.D.) were significantly faster for large color distance than for matched color $[F(1,5)=11.96, M S E=35, p=.01$, and $F(1,5)=8.04, M S E=166, p<.05$ ] (Figures 2A and $3 \mathrm{~A}$, for M.M. and A.D., respectively ), indicating facilitation by color distance. The difference between small color and matched color was not significant $(p>$ $.2, M S E=168$ and $M S E=50$, for M.M. and A.D., respectively).

To test whether the lack of difference between the small color and matched color was because of a floor
Figure 2. Mean RT in milliseconds and error percentage (in parentheses) as a function of matched color (gray), large color distance (white), and small color distance (black) for M.M. (A) and for naïve controls (B). Error bars depict 1 SEM.

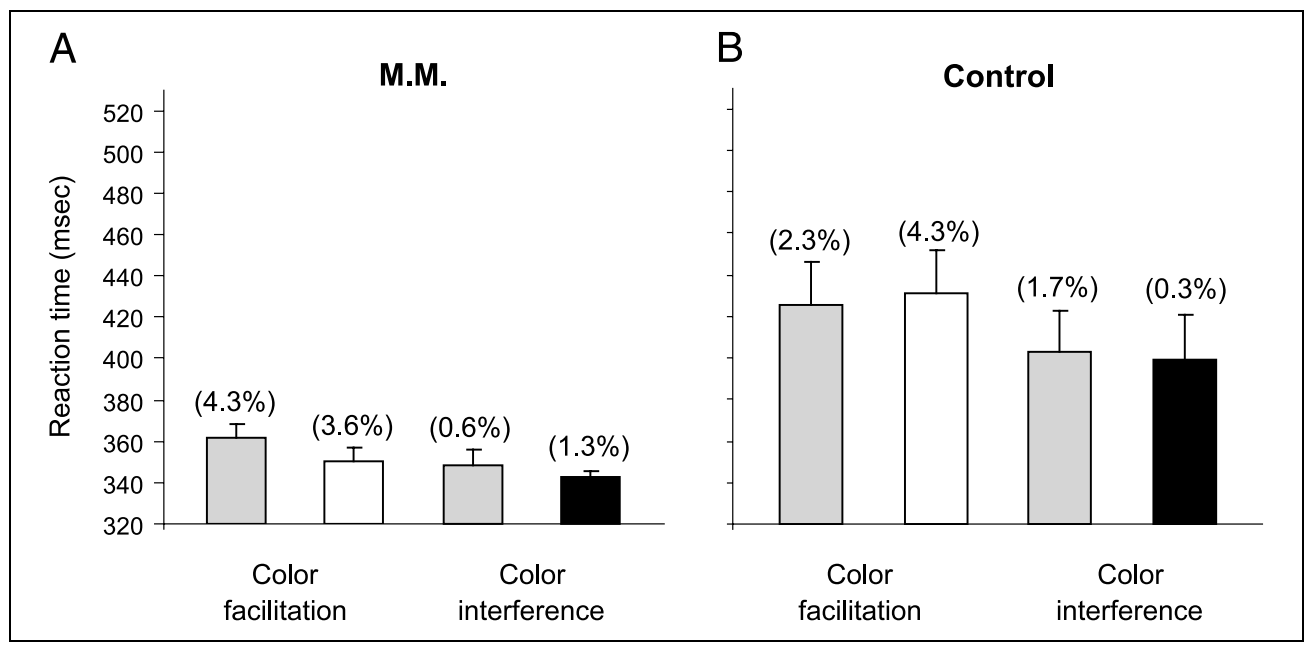


Figure 3. Mean RT in milliseconds and error percentage (in parentheses) as a function of matched color (gray), large color distance (white), and small color distance (black) for A.D.

(A) and for naïve controls

(B). Error bars depict 1 SEM.

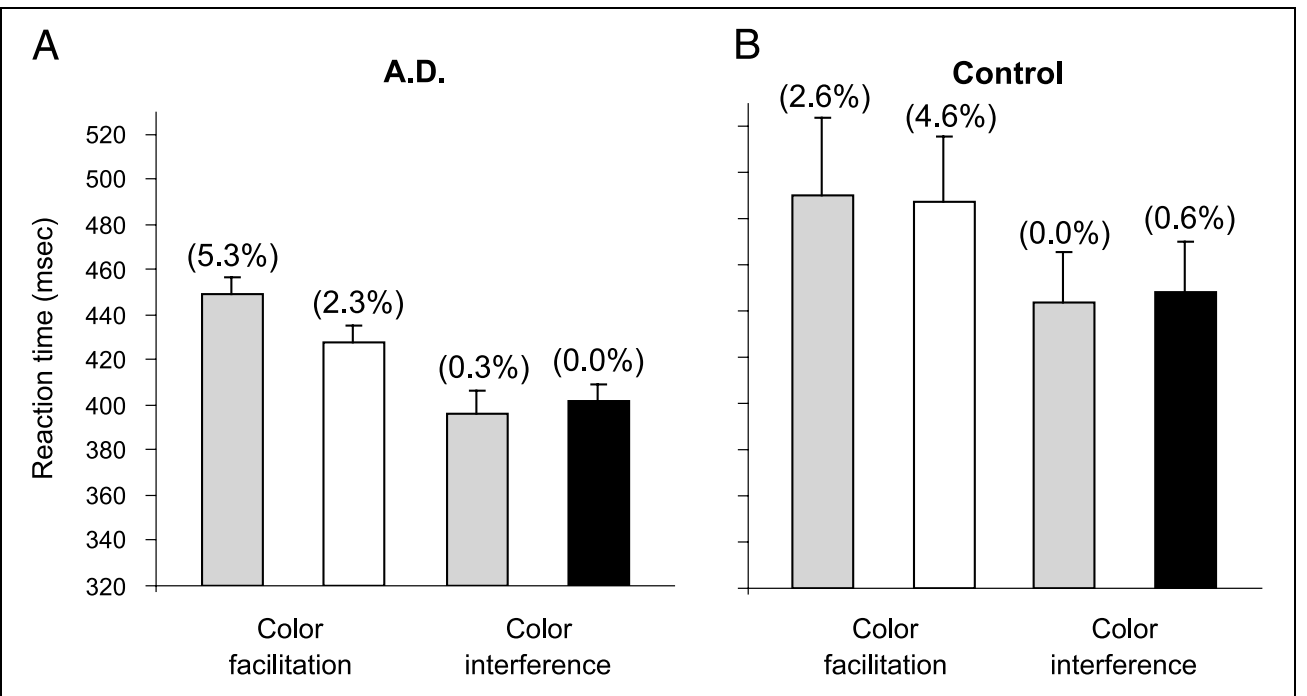

effect, we reanalyzed the data for the top $50 \%$ of the reaction time (RT) distribution of the synesthetes in each condition. As a result, RTs in both conditions increased up to a similar level of the color facilitation conditions. There was still no color congruity effect (all $p>3$ ).

\section{Naïve Control Group}

The two control groups exhibited no effect of color on RT $(p>.2$, Figures $2 \mathrm{~B}$ and $3 \mathrm{~B}, M S E=28$ and 189 for M.M.'s controls and $M S E=38$ and 130 for A.D.'s controls, for color facilitation and color interference, respectively). Examination of performance of individual participants showed no similarity to the pattern exhibited by the synesthetes.

\section{Learning Group}

The data from the learning phase (Figure 4) were modeled using a power law function (Newell \& Rosenbloom, 1981, see Methods). This function explained $97.8 \%$ of

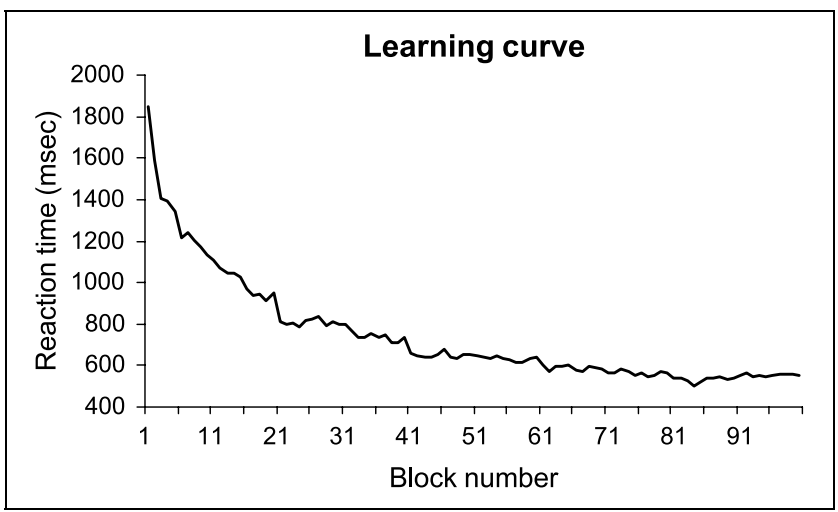

Figure 4. Mean RT in milliseconds for the learning group as a function of learning. Number of block ( $x$-axis) indicates learning block. the variance in the learning data, indicating a high degree of learning.

After completing the learning phase, the participants performed the color congruity experiment. The learning group showed the opposite pattern to the synesthetes (Figure 5). Interestingly, they responded significantly more slowly for large color distance than for matched color $[F(1,5)=6.86, M S E=46, p<.05]$. Thus, interference occurred when the color did not match the digit it was associated with. The RT difference between small color and matched color was not significant $(p>.1, M S E=45){ }^{1}$

\section{Individual Differences}

A visual inspection of the RTs gives the impression that the two synesthetes were faster than the other groups.

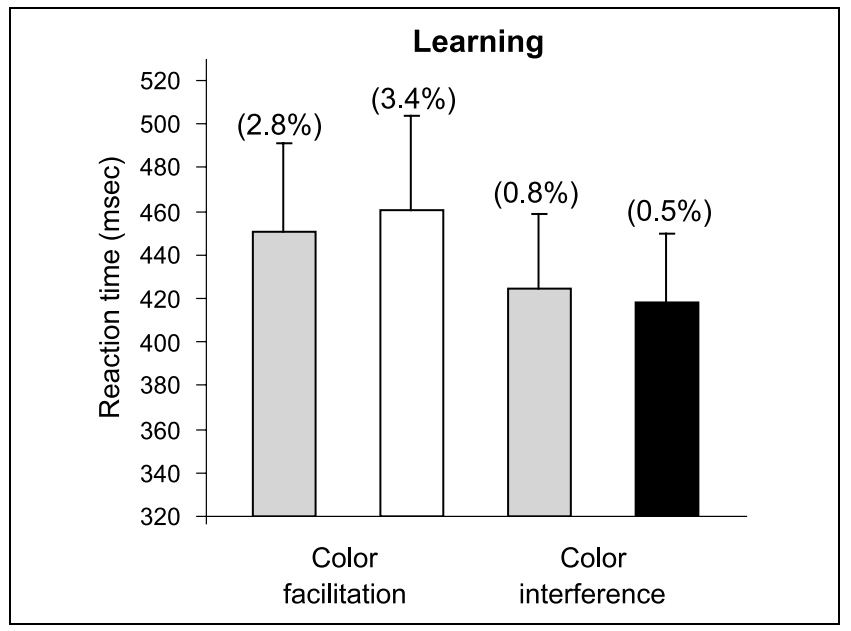

Figure 5. Mean RT in milliseconds and error percentage (in parentheses) as a function of matched color (gray), large color distance (white), and small color distance (black) for the learning group. Error bars depict 1 SEM. 
Although this might be because of different reasons, such as different amount of experience that the synesthetes had in RT studies, we tested whether these difference were statistically significant. We compared the groups using the following $t$ test:

$$
T=\left(X_{\text {synesthete }}-X_{\text {control }}\right) / S D_{\text {control }}
$$

None of the results reached significance (all $p s>.2$ ).

\section{DISCUSSION}

These results provide important new insight into the way digits and colors are processed by synesthetes. Synesthetes were faster to perform number comparison if the colors in which the numbers were presented were associated with numbers that were further apart on the mental number line (Dehaene, Bossini, \& Giraux, 1993). This indicates that the visual experience of color can influence numerical cognition in synesthetes, despite the absence of a corresponding perceptual experience. Note that facilitation in the large color condition was observed in synesthetes although digit colors were incongruent with the synesthetic colors. Obviously, when color judgments (rather than numerical judgments) are required, synesthetes are slower to respond to incongruently colored graphemes (Palmeri et al., 2002; Mattingley et al., 2001; Dixon et al., 2000; Mills et al., 1999; Odgaard et al., 1999). This has been found with M.M. and A.D. as well when they were required to respond based on color (Sagiv \& Robertson, 2004; Cohen Kadosh \& Henik, in press), namely, both synesthetes exhibited in other studies the standard Strooplike interference.

On the other hand, we did not observe interference in the small color distance condition in any of the groups. The difference in relative speed of processing the two dimensions provides a plausible explanation (Schwarz \& Ischebeck, 2003). According to the relative speed account, the large numerical distance (e.g., five units) would induce fast numerical processing, and consequently, processing of the color (distance of one unit) would not be fast enough to have an effect. Support for this explanation is provided by our recent work showing that M.M.'s color interference was modulated by the relevant numerical distance. With increase in numerical distance, color interference diminished and almost disappeared (Cohen Kadosh \& Henik, in press).

The fact that the control group that learned colornumber associations did not show similar facilitation contradicts the view that the synesthetes' performance might have been an effect of associative learning (Elias et al., 2003). Recent studies have shown that similar behavioral patterns in trained control groups and syn- esthetes were associated with different brain activity patterns (Elias et al., 2003; Nunn et al., 2002). In the present study, color automatically induced an implicit sense of magnitude that affected explicit numerical performance only in synesthetes. In contrast, in the learning group, the associative learning of digit-color pairs led to a qualitatively different effect, namely, an interference effect rather than facilitation. However, one cannot entirely rule out the possibility that lifetime learning of such pairing leads to qualitative change in the processing of the color. It would be interesting, albeit not very practicable, to compare such a group in a longitudinal study with the synesthetes for changes both at the behavioral and the anatomical levels. Nevertheless, the effect of color on magnitude judgment in both synesthetes suggests that the observed effects are not merely the result of a learned association between digits and nominal colors. At the very least, it involves an ordinal scaling of colors according to their numerical associates. However, this scaling does not imply that synesthetes see colors differently from nonsynesthetes.

It is noteworthy that parietal activation during synesthesia is common (Elias et al., 2003) and that parietal areas are activated during numerical and a variety of physical comparisons (i.e., magnitude) in nonsynesthetes (Pinel et al., 2004; Fias et al., 2003; Cohen Kadosh et al., 2005). A recent functional magnetic resonance imaging study of the size congruity effect in nonsynesthetes (Pinel et al., 2004) suggested that the interaction between numerical values and physical sizes was mediated by a common brain area (the intraparietal sulcus) being involved in the processing of both dimensions. The effect of color on magnitude in a way similar to the classical size congruity effect might suggest a new way to uncover the mechanisms that are involved in magnitude processing. We can only speculate whether interactions at the level of a common brain area or long-range interactions within the parietal lobe (Cohen Kadosh \& Henik, in press) or between parietal and temporal lobes (Ramachandran \& Hubbard, 2001a, 2001b) underlie the interference and facilitation effects observed in synesthetes.

Earlier studies suggested that synesthesia may implicate not only sensory but also conceptual aspects of the stimulus (Grossenbacher \& Lovelace, 2001; Ramachandran \& Hubbard, 2001a; Dixon et al., 2000; Cohen Kadosh \& Henik, in press). Although the idea that synesthesia could involve conceptual levels of processing is not new, our findings demonstrate that such crossactivation is not necessarily unidirectional. However, note that this bidirectional cross-talk only manifests in the task performance of synesthetes and has no experiential parallel: Our synesthetes deny that colors automatically evoke numbers in their daily life experience in any way (i.e., they do not visualize the number or think about it, although they can retrieve the number corresponding to a particular color if necessary). Despite 
this lack of explicit reports, we were able to show objectively that magnitude representations are activated by the presentation of colors.

It is unclear whether the influence of stimulus color on magnitude representation is a parallel phenomenon to the cross-activation of color areas by numeric stimuli putatively underlying synesthetic experience. One wonders why the former is not accompanied by a vivid experience of some sort as well. Eventually, there may be both qualitative and quantitative differences in the patterns of coactivation induced in each case. Crossactivation models of synesthesia do not explain how conscious color experience arises from the cross-activation in addition to any observable behavioral effect. One could argue that the cross-activation implied by our findings might be significantly weaker, for example, than the one leading to synesthetic color experience. We speculate that this is not necessarily the case. Even if the effects are comparable at the neurophysiological level, it is not clear what the experience of magnitude would be when there is nothing to count (cf. Ramachandran \& Hubbard, 2003). One reason an effect is seen in our study could be that we engage subjects in a task that requires making numeric judgments, therefore providing an outlet for this activation to manifest.

Finally, our findings suggest that the impact of synesthesia on cognition is more extensive than one might have predicted based on the phenomenology alone. Specifically, the data support a bidirectional model of synesthesia. Further investigations are needed to clarify the relationship among conscious experience, neurophysiology, and behavioral effects in synesthesia.

\section{METHODS}

\section{Participants}

Two female color-grapheme synesthetes (M.M. and A.D., both right-handed, 25 and 30 years of age, respectively), classified as projectors (Dixon et al., 2004), 6 nonsynesthetic controls who formed the learning group (matched for age, sex, handedness, education, and field of study to one of the synesthetes), and 15 nonsynesthetic controls (naive controls, 8 subjects for one of the synesthetes and 7 for the other) were tested. All participants had normal or corrected-tonormal vision, normal reading skills, and high-schoollevel mathematical abilities.

\section{Color Congruity Task}

Participants indicated which of the two digits represented the larger magnitude. Participants were asked to respond as quickly as possible but to avoid errors and to attend only to the numerical values while ignoring the colors. The participants indicated their choices by pressing one of two keys (i.e., P or Q on the keyboard) corresponding to the side of the display with the selected member of the digit pair. A stimulus display consisted of two colored digits that appeared at the center of a gray computer screen. Gray was used as a background because no number evoked this color. The center-to-center distance between the two digits was $10 \mathrm{~cm}$, and the participants viewed the screen from a distance of $55 \mathrm{~cm}$. The stimuli subtended a vertical visual angle of $1.8^{\circ}$ and a horizontal visual angle of $0.8-1.3^{\circ}$. There were three types of pairs: large color distance, matched color, and small color distance. Large color distance and matched color (color facilitation) appeared for small numerical distance, and small color distance and matched color (color interference) appeared for large numerical distance.

The synesthetes and the learning group participated in six sessions that were run on different days. The naïve control groups participated in a single session. Each session was composed of 192 trials with 48 trials per condition. In the randomized presentation, large color distance, matched color, and small color distance appeared with a ratio of 1:2:1. Before each experimental block of trials, participants received a block of 16 practice trials that was similar to the experimental block.

Each trial began with a fixation asterisk presented for $300 \mathrm{msec}$ at the center of a computer screen. Five hundred milliseconds after the fixation point disappeared, a pair of digits appeared and remained in view until the participant pressed a key (but not for more than $5000 \mathrm{msec}$ ). A new stimulus appeared $1500 \mathrm{msec}$ after the participant's response.

\section{Learning Group}

The learning group was subjected to a training phase before the color congruity task. Participants were shown the associations between digits and colors at the beginning of the training phase. In five daily meetings, participants learned to associate certain colors with certain digits (we chose the series of associations that was reported by one of the two synesthetes). Participants saw a gray digit (1-9) at the center of a colored computer screen. The background color matched or did not match the color that would have been induced by the digit. To expose participants to the same number of trials for each digit-color combination, matching and nonmatching color conditions appeared with a ratio of 1:8. Each session was composed of 20 blocks of 81 trials. The participants pressed one of two keys to indicate if the digit and color matched or not. Mapping of keys to the match/nonmatch conditions and hand of response was randomized between sessions. An error was followed by auditory feedback, and mean RT and accuracy were presented at the end of each block. To decide whether participants reached a sufficient level of learning, their performance was plotted on a graph and 
was modeled using a power law function (Newell \& Rosenbloom, 1981):

$$
\mathrm{RT}=A+B \times(N)^{-\mathrm{c}}
$$

where $\mathrm{RT}$ represents the mean reaction time in a given block, $A$ is the asymptote of learning as $N$ increases indefinitely, $B$ is the performance in time on the first block $(N=1), N$ the number of the block and $C$ is the slope of the line (i.e., the learning rate).

In addition, we compared the learning group to a group performing a word-color matching test. Participants of this group saw the same colors, with a gray color word replacing the gray digit, and had to report whether they matched (e.g., YELLOW on a yellow background) or not (e.g., RED on a yellow background). Assuming that students were skilled readers, one can use their performance in this word-color matching task as a criterion for skilled performance. All the subjects of the learning group performed equally well or better than the word-group participants in terms of RT and accuracy. After the learning was accomplished, the participants took part in the color congruity study as explained above for the synesthetes.

\section{Acknowledgments}

We thank Prof. Josef Tzelgov for valuable suggestions concerning the learning data, Ms. Lital Banouz for running the additional learning experiment, and the two anonymous reviewers for their very instructive comments leading, among other improvements, to the considerations reported in the footnote. This work was supported by a fellowship to R.C.K. from the Kreitman Foundation.

Reprint request should be sent to Roi Cohen Kadosh, Department of Behavioral Sciences, Ben-Gurion University of the Negev, Beer-Sheva, Israel, or via e-mail: roico@bgu.ac.il.

\section{Note}

1. One might argue that because the correct digit-color pairs occurred equally often as each of the eight incorrect digitcolor pairs, subjects were very likely to learn to ignore or otherwise control the association between numbers and colors. This is in contrast to synesthetes who have the "correct" pairing almost every time they see a letter or number. However, the current results showed that the learning group did not learn to ignore or control the learned association, by showing an autonomous (interfering) processing of color. Nevertheless, to explicitly test this account, we trained four new subjects on the digit-color associations in the same procedure as the original learning group. The only difference was that in this group, the correct digit-color combination appeared eight times more often than any other digit-color combinations. The pattern of results matched the original learning group by showing interference. However, in this case, the results did not reach significance, probably because of the small sample $[F(1,3)=4.46, M S E=64, p=.12]$. Moreover, the error rates analysis also showed that when the digit-color pairing did not match the learned associa- tion, participants were more prone to error $[F(1,3)=9.00$, $p=.057]$.

\section{REFERENCES}

Cohen Kadosh, R., \& Henik, A. (in press). Color congruity effect: Where do colors and numbers interact in synesthesia? Cortex.

Cohen Kadosh, R., Henik, A., Rubinsten, O., Mohr, H., Dori, H., Van de Ven, V., Zorzi, M., Hendler, T., Goeble, R., \& Linden, D. E. (2005). Are numbers special? The comparison systems of the human brain investigated by fMRI. Neuropsychologia, 43, 1238-1248.

Dehaene, S., Bossini, S., \& Giraux, P. (1993). The mental representation of parity and number magnitude. Journal of Experimental Psychology: General, 122, 371-396.

Dixon, M. J., Smilek, D., Cudahy, C., \& Merikle, P. M. (2000). Five plus two equals yellow. Nature, 406, 365.

Dixon M. J., Smilek, D., \& Merikle, P. M. (2004). Not all synaesthetes are created equal: Projector versus associator synaesthetes. Cognitive, Affective, \& Behavioral Neuroscience, 4, 335-343.

Elias, L. J., Saucier, D. M., Hardie, C., \& Sarty, G. E. (2003). Dissociating semantic and perceptual components of synaesthesia: Behavioural and functional neuroanatomical investigations. Cognitive Brain Research, 16, 232-237.

Fias, W., Lammertyn, J., Reynvoet, B., Dupont, P., \& Orban, G. A. (2003). Parietal representation of symbolic and nonsymbolic magnitude. Journal of Cognitive Neuroscience, 15, 1-11.

Fias, W., Lauwereyns, J., \& Lammertyn, J. (2001). Irrelevant digits affect feature-based attention depending on the overlap of neural circuits. Cognitive Brain Research, 12, 415-423.

Galton, F. (1880). Visualised numerals. Nature, 21, 252-256.

Grossenbacher, P. G., \& Lovelace, C. T. (2001). Mechanisms of synesthesia: Cognitive and physiological constraints. Trends in Cognitive Sciences, 5, 36-41.

Henik, A., \& Tzelgov, J. (1982). Is three greater than five: The relation between physical and semantic size in numerical comparison. Memory \& Cognition, 10, 389-395.

Lammertyn, J., Fias, W., \& Lauwereyns, J. (2002). Semantic influences on feature-based attention due to overlap of neural circuits. Cortex, 38, 878-882.

MacLeod, C. M. (1991). Half a century of research on the Stroop effect: An integrative review. Psychological Bulletin, 109, 163-203.

Mattingley, J. B., Rich, A. N., Yelland, G., \& Bradshaw, J. L. (2001). Unconscious priming eliminates automatic binding of color and alphanumeric form in synaesthesia. Nature, 410, 580-582.

Mills, C. B., Boteler, E. H., \& Oliver, G. K. (1999). Digit synaesthesia: A case study using a Stroop-type test. Cognitive Neuropsychology, 16, 181-191.

Moyer, R. S., \& Landauer, T. K. (1967). Time required for judgment of numerical inequality. Nature, 215 , 1519-1520.

Newell, A., \& Rosenbloom, P. (1981). Mechanisms of skill acquisition and the law of practice. In J. R. Anderson (Ed.), Cognitive skills and their acquisition (pp. 1-55). Hillsdale, NJ: Erlbaum.

Nunn, J. A., Gregory, L. J., Brammer, M., Williams, S. C. R., Parslow, D. M., Morgan, M. J., Morris, R. G., Bullmore, E. T., Baron-Cohen, S., \& Gray, J. A. (2002). Functional magnetic resonance imaging of synesthesia: Activation of V4/V8 by spoken words. Nature Neuroscience, 5 , $371-375$. 
Odgaard, E. C., Flowers, J. H., \& Bradman, H. L. (1999). An investigation of the cognitive and perceptual dynamics of a colour-digit synaesthete. Perception, 28, 651-664.

Palmeri, T. J., Blake, R., Marois, R., Flanery, M., \& Whestsell, W. J. (2002). The perceptual reality of synesthetic colors. Proceedings of the National Academy of Sciences, U.S.A., 99, 4127-4131.

Pinel, P., Piazza, M., Le Bihan, D., \& Dehaene, S. (2004). Distributed and overlapping cerebral representations of number, size, and luminance during comparative judgments. Neuron, 41, 983-993.

Ramachandran, V. S., \& Hubbard, E. M. (2001a). Psychological investigations into the neural basis of synaesthesia. Proceedings of the Royal Society of London, Series B, 268, 979-983.

Ramachandran, V. S., \& Hubbard, E. M. (2001b). Synaesthesia: A window into perception, thought and language. Journal of Consciousness Studies, 8, 3-34.

Ramachandran, V. S., \& Hubbard, E. M. (2003). The phenomenology of synaesthesia. Journal of Consciousness Studies, 10, 49-57.

Sagiv, N. (2004). Synesthesia in perspective. In L. C. Robertson \& N. Sagiv (Eds.), Synesthesia: Perspectives from cognitive neuroscience (pp. 3-10). New York: Oxford University Press.

Sagiv, N., \& Robertson, L. C. (2004). Synesthesia and the binding problem. In L. C. Robertson \& N. Sagiv (Eds.), Synesthesia: Perspectives from cognitive neuroscience (pp. 90-107). New York: Oxford University.

Schwarz, W., \& Ischebeck, A. (2003). On the relative speed account of the number-size interference in comparative judgment of numerals. Journal of Experimental Psychology: Human Perception and Performance, 29, 507-522.

Smilek, D., Dixon, M. J., Cudahy, C., \& Merikle, P. M. (2001). Synaesthetic photisms influence visual perception. Journal of Cognitive Neuroscience, 13, 930-936.

Stroop, J. R. (1935). Studies of interference in serial verbal reactions. Journal of Experimental Psychology, 18, 643-662. 